\title{
Potentiation of TRAIL killing activity by multimerization through isoleucine zipper hexamerization motif
}

\author{
Ji Hye Han ${ }^{1}$, Ae Ran Moon ${ }^{1}$, Jeong Hwan Chang ${ }^{2}$, Jeehyeon Bae ${ }^{4}$, Jin Myung Choi ${ }^{3}$, Sung Haeng Lee ${ }^{3}$ E Tae-Hyoung Kim ${ }^{1, *}$ \\ Departments of ${ }^{1}$ Biochemistry, ${ }^{2}$ Surgery, ${ }^{3}$ Cellular and Molecular Medicine, Chosun University School of Medicine, Gwangju 61452 , \\ ${ }^{4}$ School of Pharmacy, Chung-Ang University, Seoul 06974, Korea
}

Tumor necrosis factor (TNF)-related apoptosis-inducing ligand (TRAIL) is a homo-trimeric cytotoxic ligand. Several studies have demonstrated that incorporation of artificial trimerization motifs into the TRAIL protein leads to the enhancement of biological activity. Here, we show that linkage of the isoleucine zipper hexamerization motif to the N-terminus of TRAIL, referred as ILz(6):TRAIL, leads to multimerization of its trimeric form, which has higher cytotoxic activity compared to its native state. Size exclusion chromatography of ILz(6):TRAIL revealed possible existence of various forms such as trimeric, hexameric, and multimeric (possibly containing one-, two-, and multi-units of trimeric TRAIL, respectively). Increased number of multimerized ILz(6):TRAIL units corresponded with enhanced cytotoxic activity. Further, a high degree of ILz(6):TRAIL multimerization triggered rapid signaling events such as activation of caspases, tBid generation, and chromatin condensation. Taken together, these results indicate that multimerization of TRAIL significantly enhances its cytotoxic activity. [BMB Reports 2016; 49(5): 282-287]

\section{INTRODUCTION}

Tumor necrosis factor (TNF)-related apoptosis-inducing ligand (TRAIL) was originally identified as a member of the TNF family that can induce apoptosis in various cancer cells $(1,2)$. TRAIL mediated apoptotic induction in most transformed tumor cells occurs by stimulation of its cognate receptors DR4 (or TRAILR1) and DR5 (or TRAILR2). However, TRAIL related induction of apoptosis in normal cells is known to be limited by a yet unknown mechanism. The active form of TRAIL is a homo-trimer that triggers trimerization of DR4 or DR5 and

${ }^{*}$ Corresponding author. Tel: +82-62-230-6294; Fax: +82-62-2264165; E-mail: thkim65@chosun.ac.kr

http://dx.doi.org/10.5483/BMBRep.2016.49.5.245

Received 25 November 2015, Revised 9 December 2015, Accepted 11 December 2015

Keywords: Apoptosis, Cell death, Isoleucine zipper, Multimerization, TRAIL subsequent recruitment of FADD and caspase- 8 to form the death-inducing signaling complex (DISC) (3-5), which ultimately results in apoptotic cell death.

TRAIL is a type II transmembrane protein, which can be produced in a soluble form by protease-mediated cleavage of its extracellular region or by bacterial expression of recombinant forms that encompass its extracellular region $(1,2)$. Structural studies of TRAIL have revealed that it forms a trimer, which further bind to the trimeric DR5 protein (6-8). TRAIL can form an apoptosis-inducing trimer in its native soluble form. Researchers have tried to stabilize this trimeric state of the soluble TRAIL by fusing trimerization motifs such as the isoleucine zipper (ILz) trimerization motif $(9,10)$ or the tenascin-C-derived trimerization motif (11). Furthermore, the apoptosis-inducing activity of soluble TRAIL has been shown to be greatly enhanced by oligomerization of soluble Flag-tagged TRAIL plus Flag-antibodies (11). Thus, the trimerization of TRAIL as well as oligomerization of its trimeric form appears to be the key determinants of its apoptosis-inducing activity.

In this report, we developed a novel ILz(6):TRAIL protein containing the hexamer formation domain of the isoleucine zipper motif (which may exist in two trimeric forms) followed by an extracellular region of the native TRAIL protein (amino acids 114-281). Unexpectedly, we found that ILz(6):TRAIL can form trimers, hexamers, and various multimers. Further, we showed that the apoptosis-inducing activity of soluble TRAIL is potentiated by its multimerization.

\section{RESULTS}

\section{Generation of recombinant ILz(6):TRAIL}

TRAIL is a homo-trimeric membrane protein that is known to induce apoptosis in various tumor cells by binding to its cognate trimeric DR5 receptor. Recombinant soluble TRAIL composed of the extracellular region of TRAIL (amino acids 114281) killed tumor cells. Fusion of the trimerization motif of an isoleucine zipper motif at the $\mathrm{N}$-terminal region of TRAIL led to enhanced trimer formation $(9,10)$. Based on this we assume that owing to the presence of a pre-oligomerized state the hexameric TRAIL protein (composed of two TRAIL trimeric units, amino acids 114-281) possibly displays a strong cytotoxic activity. The addition of a cysteine residue to the trimerization 
motif of an isoleucine zipper has been shown to form a hexamer $(12,13)$. In order to generate a recombinant hexameric TRAIL protein (amino acid 114-281), the hexamerization motif of the isoleucine zipper was fused to TRAIL (114-281) at its $\mathrm{N}$-terminal region resulting in ILz(6):TRAIL (Fig. 1A). The recombinant ILz(6):TRAIL protein was expressed in E. coli BL21(DE3) and purified using a Ni-NTA His affinity column (Fig. 1B). Recombinant ILz(6):TRAIL efficiently induced cell death in HCT116 (Fig. 1C) and BJAB cells (Fig. 1D). Furthermore, ILz(6):TRAIL showed a more potent cytotoxic activity compared to the recombinant TRAIL protein (amino acids 114-281; hereafter referred as TRAIL) in BJAB cells (Fig. 1D). Additionally, the recombinant ILz(6):TRAIL showed enhanced cytotoxic activity on BJAB cells compared to recombinant TRAIL, which does not possess an isoleucine zipper hexamerization motif (Fig. 1E).

\section{Multimerization of recombinant ILz(6):TRAIL}

In order to examine the multimerization status of the recombinant proteins, ILz(6):TRAIL or TRAIL were subjected to crosslinking with Bismaleimidohexane (BMH) followed by separation on SDS-PAGE. ILZ(6):TRAIL was cross-linked more favorably compared to TRAIL (Fig. 2A), indicating that in comparison to TRAIL, ILZ(6):TRAIL was a well-formed multimer. These results, however, did not conclusively determine whether the
ILz(6):TRAIL protein existed in a trimeric or a hexameric form.

Because the N-terminus of the ILz(6) motif contains a cysteine residue, we expected that this cysteine residue may lead to the formation of disulfide bonds between the trimeric state of ILz(6):TRAIL and thereby result in single or multiple units (trimers, hexamers, nonamers, dodecamers, etc.) of the trimeric ILz(6):TRAIL. Size exclusion chromatography separated the various multimeric states of ILz(6):TRAIL, which was found to form not only trimers and hexamers, but also mega-multimers (Fig. 2B). Since trimers, hexamers, and mega-multimers were found abundantly, we concluded that depending on the conditions of purification, the three multimeric forms of ILz(6): TRAIL were interchangeable. To determine the multimeric states of mega-multimer fraction of ILz(6):TRAIL, sedimentation velocity experiments were carried out using analytical ultracentrifugation (AUC). The AUC results indicated that the fraction of ILz(6):TRAIL mega-multimers contain various multimeric forms including not only trimer and hexamer, but also 12-mer, 18-mer, 24-mer, 33-mer, and 45-mer. It is likely that the presence of cysteine residue at $\mathrm{N}$-terminus of isoleucine hexamerization motifs in ILz(6):TRAIL led to various multi-units of trimeric ILz(6):TRAIL being formed through disulfide bonds between trimeric forms of ILz(6):TRAIL as we expected (Fig. 2C). Distribution of these multimeric forms of ILz(6): TRAIL appear to be dependent on E. coli culture conditions
A

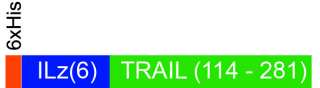

B

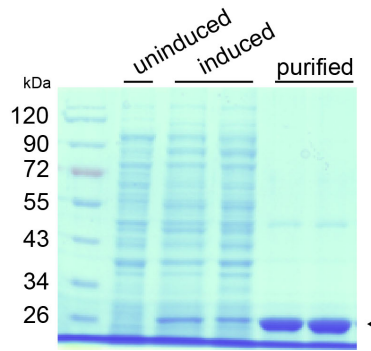

D

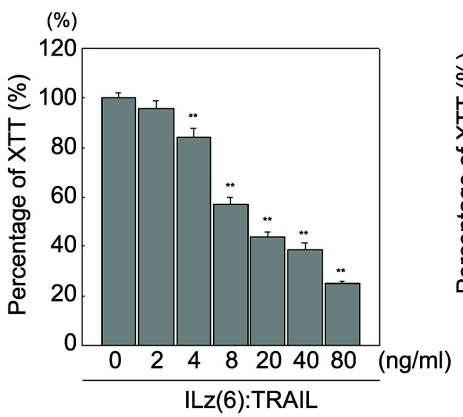

E

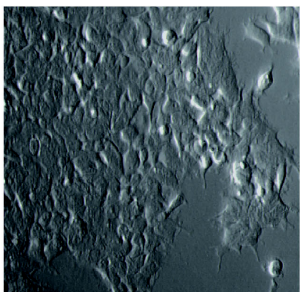

$0(\mathrm{ng} / \mathrm{ml})$

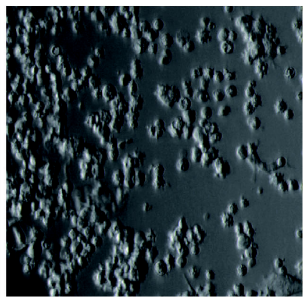

$100(\mathrm{ng} / \mathrm{ml})$
ILz(6):TRAIL

- ILz(6):TRAIL

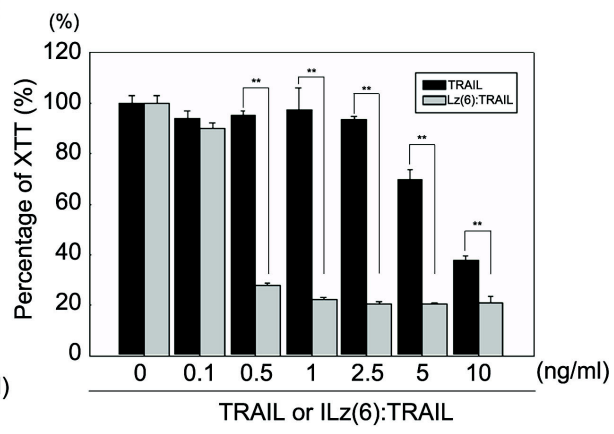

Fig. 1. Development of ILz(6):TRAIL. (A) Schematic representation of the ILz(6):TRAIL protein. The $6 x$ His-tag was essential for Ni-NTA affinity purification; ILz(6) represents the isoleucine zipper hexamerization motif and TRAIL (114-281) represents the extracellular region of human TRAIL amino acids 114-281; (B) Purification of the recombinant ILz(6):TRAIL protein. The bacterial expression vector pET23a(+):ILz(6): TRAIL was transformed into $E$. coli BL21 (DE3) cells and induced with IPTG. Recombinant ILz(6):TRAIL was purified using a Ni-NTA His-binding affinity column; (C) HCT116 cells were treated overnight with recombinant ILz(6):TRAIL following by recording light microscope images; (D) Activity analysis of recombinant ILz(6):TRAIL. $B J A B$ cells were treated with the the indicated amounts of purified recombinant ILz(6):TRAIL for 2 hours and cell death was determined using the XTT assay; (E) Comparison of the apoptosis-inducing activities of TRAIL and ILz(6):TRAIL. BJAB cells were treated with recombinant TRAIL or ILz(6): TRAIL for 18 hours and cell death was measured using the XTT assay. 
$\begin{array}{ll}\text { A } & \text { M.W } \\ (\mathrm{kD})\end{array}$

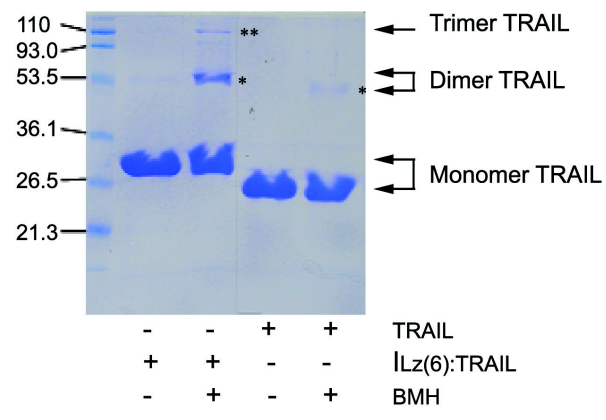

B

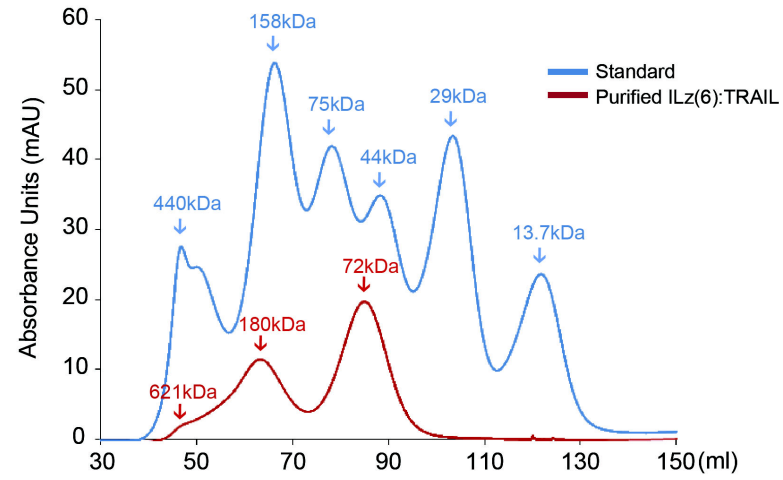

C
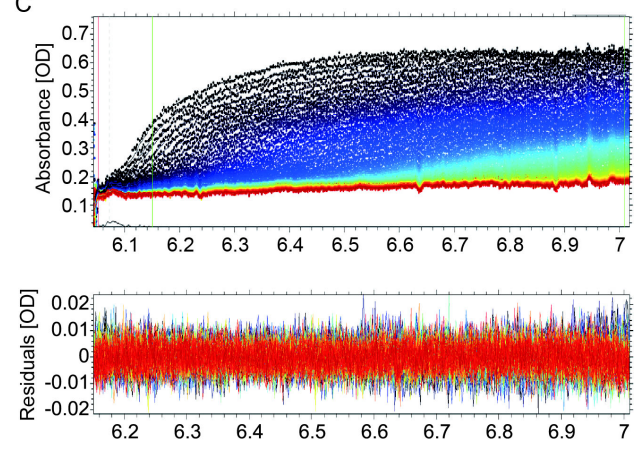

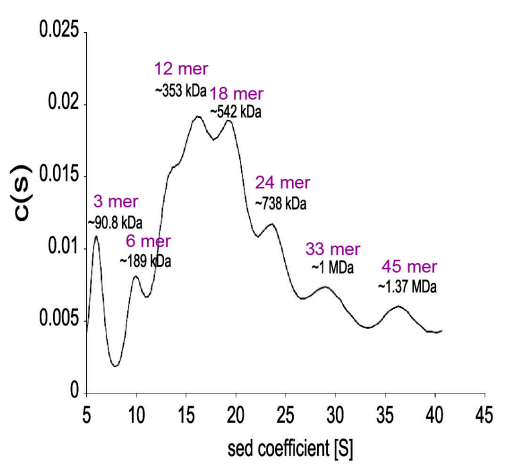

Fig. 2. Multimerization of ILz(6):TRAIL. (A) Recombinant ILz(6):TRAIL and recombinant TRAIL proteins were crosslinked with $\mathrm{BMH}$ and subjected to SDS-PAGE followed by staining with Coomassie Brilliant Blue. Monomers, dimers, and trimers of TRAIL and ILz(6):TRAIL are indicated; (B) Size fractionation of ILz(6):TRAIL. Recombinant ILz(6): TRAIL was subjected to size exclusion chromatography and the indicated relative molecular weights are comparable to molecular weight standards of dextran. (C) Multimeric form of Lz(6):TRAIL was obtained by size exclusion chromatography as described above. Sedimentation velocity AUC was performed, and AUC data were analyzed by continuous size distribution analysis using SEDFIT software (available at http://www.analyticalultracentrifugation.com). such as growth temperature and time, buffer composition, and purification temperature. However, the key factors that determine the varying multimeric states of ILz(6):TRAIL were not defined in detail.

\section{Potentiation of ILz(6):TRAIL by multimerization}

Several reports have shown that the cytotoxic activity of death ligands, including TRAIL, in various tumor cells is enhanced upon their multimerization. Thus, we expected the ILz(6):TRAIL multimer and trimer to possess the highest and lowest cytotoxic activity, respectively. Indeed, the multimer of ILz(6):TRAIL showed the highest cytotoxic activity in Jurkat,
$B J A B$, and HeLa cells and the trimer showed the least activity in these tumor cells (Fig. 3A-C). These results confirmed that multimerization of ILz(6):TRAIL potentiates its killing activity in tumor cells.

Cell death-signaling pathways were monitored at different points in order to characterize the responsiveness of multimeric forms of ILz(6):TRAIL. Multimeric ILz(6):TRAIL resulted in an obvious chromatin condensation and morphological changes in Jurkat cells within 1 hour, whereas a higher response time of either 2 or 4 hours was needed for chromatin condensation in the presence of hexameric or trimeric ILZ(6): TRAIL, respectively (Fig. 4A). Furthermore, the cleaved forms 
A

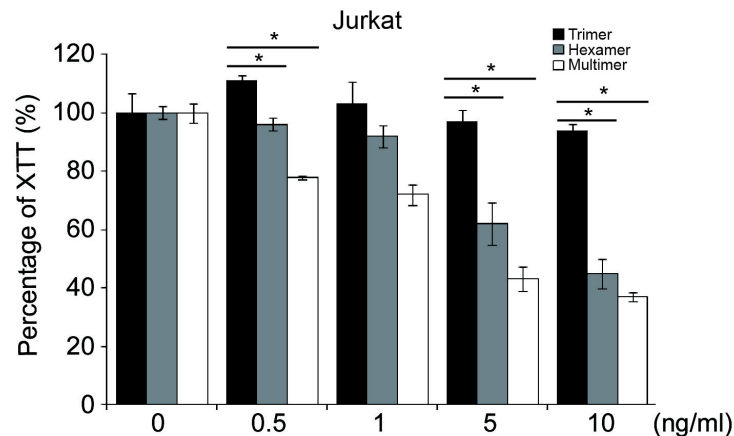

B

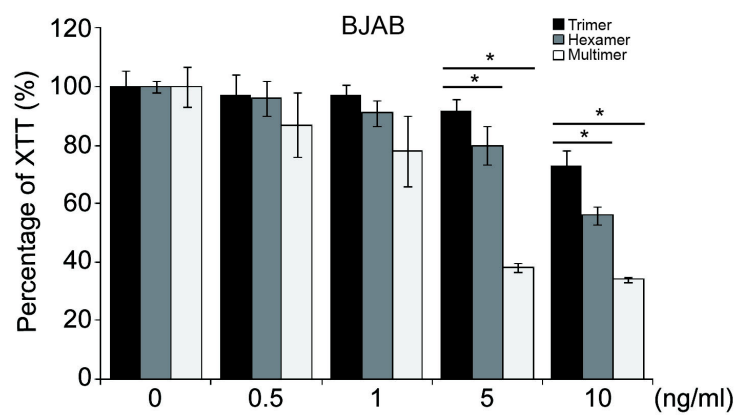

C

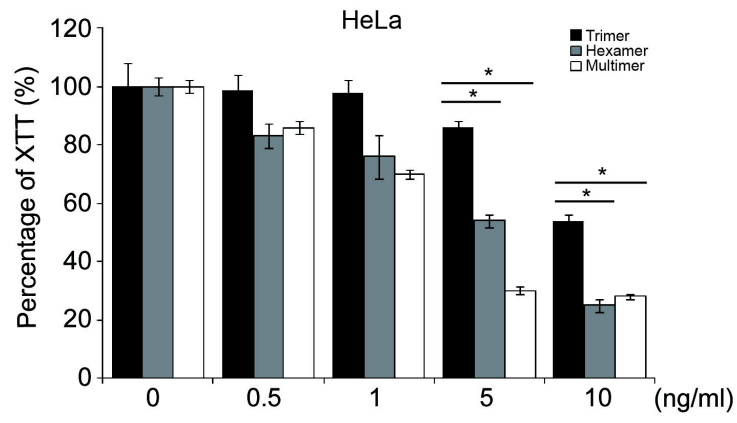

Fig. 3. ILz(6):TRAIL is a potent killer. Jurkat (A); BJAB (B); and HeLa (C) cells were treated with trimers, hexamers, or multimers of recombinant ILz(6):TRAIL and cell viability was measured by XTT assay.

of caspase-3 and caspase- 8 appeared within 30 minutes in response to multimeric ILz(6):TRAIL, but not until 60 minutes or 2 hours in response to hexameric or trimeric ILz(6):TRAIL, respectively. Multimeric ILz(6):TRAIL alone was able to generate tBid within 60 minutes after treatment. These results clearly show that multimerization of ILz(6):TRAIL enhances its cytotoxic activity.

\section{DISCUSSION}

In this study, we showed that the recombinant ILz(6):TRAIL protein possesses superior cytotoxic activity compared to re-

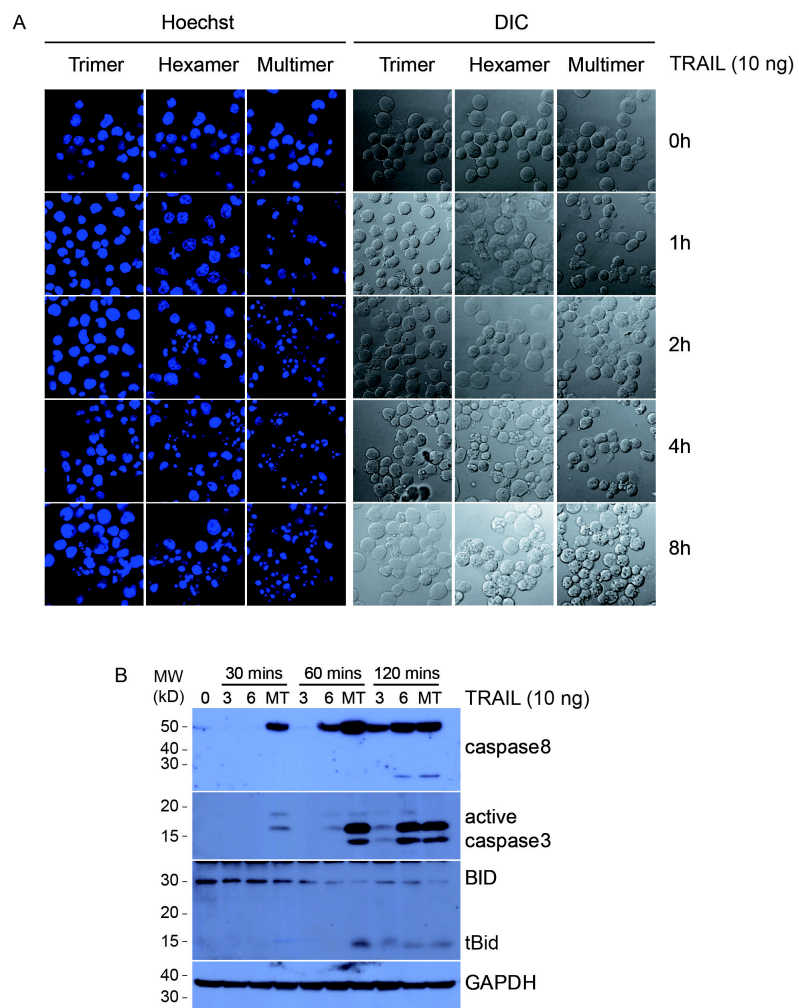

Fig. 4. ILz(6):TRAIL is a potent killer. (A) Jurkat cells were treated with trimers, hexamers, or multimers of recombinant ILz(6):TRAIL protein for indicated times and subjected to Hoechst staining. Cells were then imaged with a fluorescent or a DIC microscope. (B) Jurkat cells were treated with trimers, hexamers, or multimers (MT) of recombinant ILz(6):TRAIL protein for 30, 60, and 120 minutes. Cell lysates were then subjected to immunoblotting against caspase-8, caspase-3, Bid, and GAPDH.

combinant TRAIL. The presence of a hexamerization motif from the isoleucine zipper at the $\mathrm{N}$-terminus of TRAIL prompts not just hexamerization but also multimerization of ILz(6):TRAIL, resulting in remarkable enhancement of the cytotoxic activity in various cancer cells. Size exclusion chromatography and AUC of ILz(6):TRAIL demonstrated that it can form not only trimeric or hexameric but also various multimeric forms. We believe that the cysteine residue of the hexamerization motif in ILz(6):TRAIL leads to these various multimeric forms of ILz(6): TRAIL. We speculate that one-unit of trimeric ILz(6):TRAIL is likely to form two-units of trimeric ILz(6):TRAIL by disulfide bonding, which then accelerates the formation of various multi-units of trimeric ILz(6):TRAIL. This may be attributed to favorable disulfide bond cross-linking between one- and twounits of trimeric ILz(6):TRAIL.

Previous studies have shown that artificial trimerization of soluble trimeric ligands such as CD40L (14), GITR (15), FasL (16), and TRAIL (10) mediated by the isoleucine zipper trimeri- 
zation motif can enhance their biological activity. Consistent with previous reports, the multimeric forms of ILz(6):TRAIL showed superior cytotoxic activities compared to their lower multimeric counterparts. The multimerization of ILz(6):TRAIL also resulted in rapid signaling events such as activation of caspases, tBid generation, and chromatin condensation. Although the exact reason and mechanism associated with high cytotoxicity of multimeric ILz(6):TRAIL forms is not clear, we speculate that a pre-multimerized ILz(6):TRAIL could facilitates the multimerization of DR5 on the cell surface. The facilitated multimerization of DR5 on the cell surface by multimeric ILz(6):TRAIL could, in turn, ease to form the death-inducing signaling complex (DISC) and/or to increase the stability of DISC complex in the cytoplasmic side of DR5, ultimately resulting in the early activation of caspase-8.

Taken together, our results indicate that the multimerization of ILZ(6):TRAIL can potentiate the biological activity of TRAIL by accelerating the induction of cell death signaling pathways.

\section{MATERIALS AND METHODS}

\section{Construction of ILz(6):TRAIL expression vectors}

cDNA, encoding amino acids $114-281$ of human TRAIL, was amplified by reverse transcriptase - polymerase chain reaction (RT-PCR) using total RNA isolated from HeLa cells. The following primers were used: hTRAIL(114):Sma1 (GGGCCCGGGGT GAGAGAAAGAGGTCCTCAG) and hTRAIL(281):EcoR1 (CCC GAATTCTTAGCCAACTAAAAAGGCCC). The amplified TRAIL cDNA was cloned into a modified version of pET23a(+) bearing a $6 \mathrm{xHis}$ tag at the $5^{\prime}$ end of the multi cloning site. The synthetic oligonucleotide encoding the isoleucine hexamerization motif (ATGTGCGGAGGAAAGCAAATCGAAGACAAGATAGA AGAAATCCTATCAAAGATCTACCACATCGAAAACGAGATC GCACGAATCAAGAAGCTAATCGGAGAA) was cloned into the Smal and EcoRV sites of the modified pET23a(+) vector, whereas the PCR-amplified DNA, encoding human TRAIL amino acids114 - 281, was cloned into pET23a(+):ILz(6) at EcoRV and BamHI sites and has been referred to as pET23a(+): ILZ(6):TRAIL.

\section{Purification of recombinant TRAIL or ILz(6):TRAIL}

E. coli BL21 (DE3) cells were transformed with pET23a(+): TRAIL or pET23a(+):ILz(6):TRAIL vectors and cultured in LB media at $28^{\circ} \mathrm{C}$ for 2 days in the presence of IPTG. Cells were then harvested by centrifugation $\left(5,000 \times \mathrm{g}\right.$ for $5 \mathrm{~min}$ at $\left.4^{\circ} \mathrm{C}\right)$ and protein purification was performed using Ni-NTA His binding resins (Novagen, Carlsbad, CA, USA), according to the manufacturer's instructions.

\section{Size exclusion chromatography}

The purified recombinant ILz(6):TRAIL proteins were put through a Sephacryl-S200 size exclusion chromatography column (GE Healthcare Life Sciences, Cat. No. 17-0584-01). The column was operated with an AKTA-Prime Plus Liquid
Chromatography System (GE Healthcare Life Sciences, Sweden). The total volume of the collected fractions was $109.9 \mathrm{ml}$ and the void volume was $39.8 \mathrm{ml}$.

\section{Analytical ultracentrifugation (AUC)}

A multimeric form of Lz(6):TRAIL was obtained by size exclusion chromatography as described above, and was dialysed in buffer containing $20 \mathrm{mM}$ Tris-Cl pH 7.5 and $50 \mathrm{mM} \mathrm{NaCl}$ for analytical ultracentrifugation. Sedimentation velocity AUC experiments were performed at $20^{\circ} \mathrm{C}$ using a Beckman XL-A ultracentrifuge (Palo Alto, CA, USA) using an AN-60 Ti fourhole rotor. Absorbance scans were taken at 30,000 rpm for 72 hrs. AUC data were analyzed by continuous size distribution analysis using SEDFIT software (available at www.analyticalultracentrifugation.com).

\section{Cell culture and XTT assay}

$B J A B$ lymphoma cells (a generous gift from Genentech, San Francisco, CA, USA) were cultured in phenol red free RPMI medium (Welgene, Daegu, S. Korea) containing 10\% fetal bovine serum, 10 units $/ \mathrm{ml}$ penicillin, and $1 \mathrm{mg} / \mathrm{ml}$ streptomycin (all purchased from Gendepot, Barker, TX, USA). For the cell killing activity assay, BJAB cells $\left(1.5 \times 10^{4}\right.$ cells $\left./ \mathrm{ml}\right)$ were plated and treated with recombinant TRAIL or ILZ(6):TRAIL. XTT solution (Promega, Madison, WI, USA) was then added and absorbances were monitored at $493 \mathrm{~nm}$ using a TECAN Infinite M200 monochromator (Tecan, Mannedorf, Switzerland).

\section{Statistical analysis}

Results are reported as means \pm SD. The Student's t test was used to evaluate differences between more than two groups. Statistical significance was accepted for $\mathrm{P}$ values $<0.05$.

\section{ACKNOWLEDGEMENTS}

This research was supported by the Basic Science Research Program through the National Research Foundation of Korea funded by the Ministry of Education, Science and Technology (Grant number 2010-0009201 and NRF-2013R1A1A2058254 to TH Kim), by the Ministry of Science, ICT, and Future Planning (NRF-2014R1A2A1A11050442 to TH Kim), and by the National R \& D Program for Cancer Control funded by the Ministry of Health and Welfare of the Korean government (Grant number 1020100 to TH Kim). This study was also supported by grants (to JH Chang, Y2011) from the Clinical Medicine Research Institute at Chosun University Hospital.

\section{REFERENCES}

1. Wiley SR, Schooley K, Smolak PJ et al (1995) Identification and characterization of a new member of the TNF family that induces apoptosis. Immunity 3, 673-682

2. Pitti RM, Marsters SA, Ruppert S, Donahue CJ, Moore A 
and Ashkenazi A (1996) Induction of apoptosis by Apo-2 ligand, a new member of the tumor necrosis factor cytokine family. J Biol Chem 271, 12687-12690

3. Chaudhary PM, Eby M, Jasmin A, Bookwalter A, Murray J and Hood L (1997) Death receptor 5, a new member of the TNFR family, and DR4 induce FADD-dependent apoptosis and activate the NF-kappaB pathway. Immunity 7, 821-830

4. Schneider P, Thome M, Burns K et al (1997) TRAIL receptors 1 (DR4) and 2 (DR5) signal FADD-dependent apoptosis and activate NF-kappaB. Immunity 7, 831-836

5. Sheridan JP, Marsters SA, Pitti RM et al (1997) Control of TRAIL-induced apoptosis by a family of signaling and decoy receptors. Science 277, 818-821

6. Hymowitz SG, Christinger HW, Fuh G et al (1999) Triggering cell death: the crystal structure of Apo2L/TRAIL in a complex with death receptor 5 . Mol Cell 4, 563-571

7. laccarino G, Smithwick LA, Lefkowitz RJ and Koch W] (1999) Targeting Gbeta gamma signaling in arterial vascular smooth muscle proliferation: a novel strategy to limit restenosis. Proc Natl Acad Sci U S A 96, 3945-3950

8. Mongkolsapaya J, Grimes JM, Chen N et al (1999) Structure of the TRAIL-DR5 complex reveals mechanisms conferring specificity in apoptotic initiation. Nat Struct Biol 6, 1048- 1053

9. Jeong M, Kwon YS, Park SH et al (2009) Possible novel therapy for malignant gliomas with secretable trimeric
TRAIL. PloS ONE 4, e4545

10. Walczak H, Miller RE, Ariail K et al (1999) Tumoricidal activity of tumor necrosis factor-related apoptosis-inducing ligand in vivo. Nat Med 5, 157-163

11. Berg D, Stuhmer T, Siegmund D et al (2009) Oligomerized tumor necrosis factor-related apoptosis inducing ligand strongly induces cell death in myeloma cells, but also activates proinflammatory signaling pathways. FEBS Lett 276 , 6912-6927

12. O'Shea EK, Rutkowski R, Stafford WF 3rd and Kim PS (1989) Preferential heterodimer formation by isolated leucine zippers from fos and jun. Science 245, 646-648

13. Harbury PB, Zhang T, Kim PS and Alber T (1993) A switch between two-, three-, and four-stranded coiled coils in GCN4 leucine zipper mutants. Science 262, 1401-1407

14. Morris AE, Remmele RL Jr, Klinke R, Macduff BM, Fanslow WC and Armitage RJ (1999) Incorporation of an isoleucine zipper motif enhances the biological activity of soluble CD40L (CD154). J Biol Chem 274, 418-423

15. Cui D, Wang S, Chen Y et al (2010) An isoleucine-zipper motif enhances costimulation of human soluble trimeric GITR ligand. Cell Mol Immunol 7, 316-322

16. Shiraishi T, Suzuyama K, Okamoto H et al (2004) Increased cytotoxicity of soluble Fas ligand by fusing isoleucine zipper motif. Biochem Biophys Res Commun 322, 197202 